\title{
The Effect Of Mobile Phone Use While Driving On Response Time: Driving Simulator Study
}

\author{
Hussein S. Mutar*, Ahmed S. Abduljabbar, Ammar A. Mohammed $\mathbb{C}$ \\ University of Technology-Iraq, Alsina'a street, 10066 Baghdad, Iraq. \\ *Corresponding author Email: bce.19.28@grad.uotechnology.edu.iq
}

\section{H I G H L I G H T S}

- Using driving simulator to assess mobile phones distraction on driving performance.

- The response time is increased while using smart phones while driving.

- The results indicate that the response time increases with task difficulty.

- Young participants are less affected when compared with older people.

\section{A R T I C L E I N F O}

Handling editor: Wasan I. Khalil

\section{Keywords:}

Driving Simulator

Mobile Phone Use Hand-Held Mobile

Hands-Free Mobile

Reading text messages

Sending text messages

\author{
A B S T R A C T
}

Mobile phone use is one of the most common daily tasks and this is normal, however, this task could be problematic while driving. The use of mobile phones while driving has become a major cause of road accidents and poses a threat to public health. This study investigated the effect of mobile phone usage while driving on response time, as it investigated four mobile phone tasks (hands-free calling, hand calls, reading text messages, and sending text messages) in addition to basic driving. A total of 42 participants, ranging in age from (19 to 55), with a mean age (mean $=33.14, \mathrm{SD}=10.26$ ) participated in the driving simulation at the University of Technology and all participants performed five tasks. The participants had to interact with voice commands by performing the throttle maneuver. The results concluded with a delay in response, which means an increase in cognitive reaction time when using a mobile phone compared to basic driving. It has also been found that the response time increases with the age of drivers.

\section{Introduction}

Using a mobile phone while driving has become very common and is one of the main causes of traffic accidents all over the world $[1,2]$. With the great advancement in the field of communication technologies installed in cars or portable that can be introduced to vehicles, mobile phones are receiving great attention concerning their direct impact on driving performance and safety for road users[3]. Although most countries banned the use of mobile phones while driving a car, many drivers still use it while driving. The mobile phone contributes to the dispersion of drivers and distraction from basic driving tasks. In the United States of America, a study (number $=3265$ ) found that $48 \%$ of dispersed drivers were caused by their distraction because they were busy with the mobile phone while driving[4]. According to the World Health Organization (WHO)[5], the percentage of people who text while driving is still very high around the world: In the United States, the United Kingdom, and Australia, respectively, 27 percent, 45 percent, and 16.67 percent. And according to Lambert and Regan[6] More than half of drivers admitted to sending or reading (1-5) text messages a week while driving. The increased use of mobile phones while driving has increased the risk of car accidents, as the increase in traffic accidents is closely linked to the increase in mobile phone users while driving. For example, according to the [7]NHTSA (National Highway Traffic Safety Administration, 2019), cell phones were involved in 401 fatal accidents in the United States in 2017 (14 percent of all fatal distraction-affected crashes). With reference to[2] the World Health Organization (WHO) report, drivers who use their cell phones while driving are four times more likely to have accidents than drivers who do not use their cell phones while driving. In general, many studies have been conducted on the effect of mobile phone use on driver performance, some for calls and others for text messages. This paper deals with a hypothetical examination of a number of drivers using a driving simulator to find out the effect that using a mobile phone for hand-held calls, hands-free calls, reading and sending text messages, on the response time of drivers as a result of the distraction caused by the distraction of the mobile phone. According to a study[8], talking on the 
phone while driving causes a drop in condition comprehension and a pause in reacting to incidents that arise in the driving world, which may lead to incidents. One of the most useful metrics for assessing the impact of distraction on event detection efficiency is reaction time. The four types of mobile phone diversion using on are simple chat, complicated conversation, easy text messaging, and complex text writing. In the pedestrian crossing task, the tasks increased response times by $40 \%, 95 \%, 137$ $\%$, and $204 \%$, respectively. The activities caused response times to rise by $48 \%, 65 \%, 121 \%$, and $171 \%$ when the road crossing was caused by parked cars. As a result, all phone use conditions were found to be the most important factors in driving efficiency degradation. According to[9] Drivers are distracted by cell phone conversations so their attention is diverted away from the primary driving activity. As a result, the response times of drivers have been studied in different study settings, including laboratory, driving simulator, and in-field experiments, as a surrogate indicator of the crash probability of cell phone distraction It was found that the drivers' reaction times were $40 \%$ longer in the dispersion condition compared to the baseline (non-dispersed). Referring to the study [10] they examined the braking performance of drivers who were distracted using a mobile phone using a laboratory station that simulated the performance of the foot when driving. 22 participants were asked to stop as soon as possible when the red lamp was activated in the laboratory and found that both hands-free and mobile phone conversations by hand-held, Conversation on a mobile phone slowed Reaction Time according to the findings obtained.[11] Investigated distracted drivers' reaction times in the peripheral detection task (PDT) under different environmental complications and found that, regardless of phone form or environmental complexities, the PDT response time was longer, and precision was lower in cell phone conditions. Several studies in Iraq dealt with traffic issues and traffic transportation problems, such as $[12,13,14]$. However, they did not address the distracted behavior of drivers using mobile phones.

\section{Materials and Methods}

\subsection{Participants}

A total of (42) participants participated in the study of the effect of using a mobile phone while driving on cognitive reaction time. The average years of experience for the participants (mean $=9.83$ years, $\mathrm{SD}=8.44$ ), and the rate of accidents committed (mean $=1.45, \mathrm{SD}=1.38)$. As shown in Figure (1).

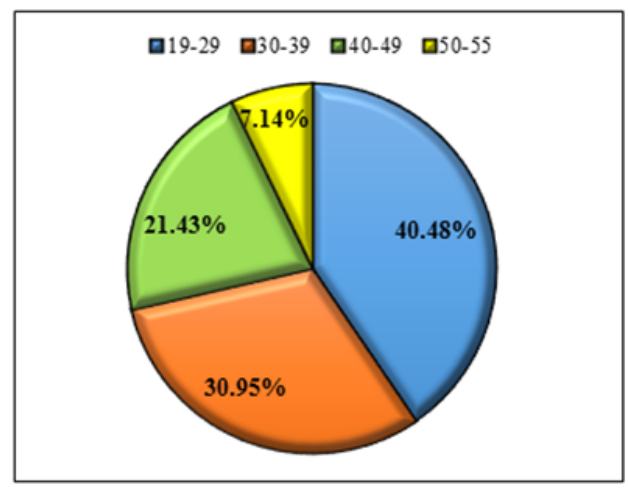

Figure 1: Participants by age categories

\subsection{Apparatus}

\subsubsection{Driving simulator}

A driving simulator located in the Civil Engineering Department of the University of Technology was used. This device consists of a group of parts that simulate its siblings in real vehicles such as (cockpit, transmission, brakes, throttle) and three screens linked to each other to give an integral view, where the front screen represents the vehicle's windscreen, and the two side screens represent the side glass on the right and left the driver is in real vehicles. The device gives an immersive and exciting virtual driving experience and has a surround sound that gives a feeling of real driving. Figure (2) illustrates the used driving simulator.

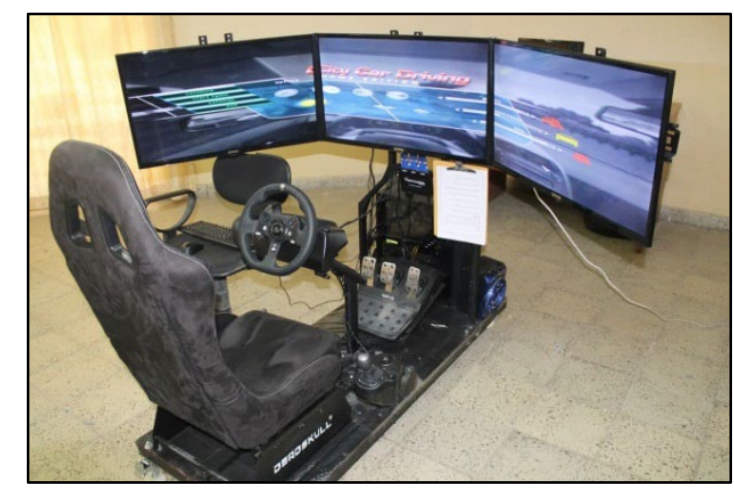

Figure 2: Driving simulator device 


\subsubsection{Test scenario}

In this study, the highway environment was adopted for examination, where the examination path forms a straight road with a length of (20) $\mathrm{km}$ with some horizontal curves and the presence of one vertical curve in the way of going and the other in the return. As it divides the road into (10) km going and (10) km back. The highway consists of three lanes, each of which is 3.5 meters wide. After testing the different traffic densities by the researcher, a medium and lower traffic density was selected in the examination because it is more suitable and smoother with the mobile phone impact tests. Can you define different levels of traffic or density here areas adjacent to the highway have car maintenance buildings, football fields, and large billboards that give a realistic driving feel. Figure (3) shows the course of the test.

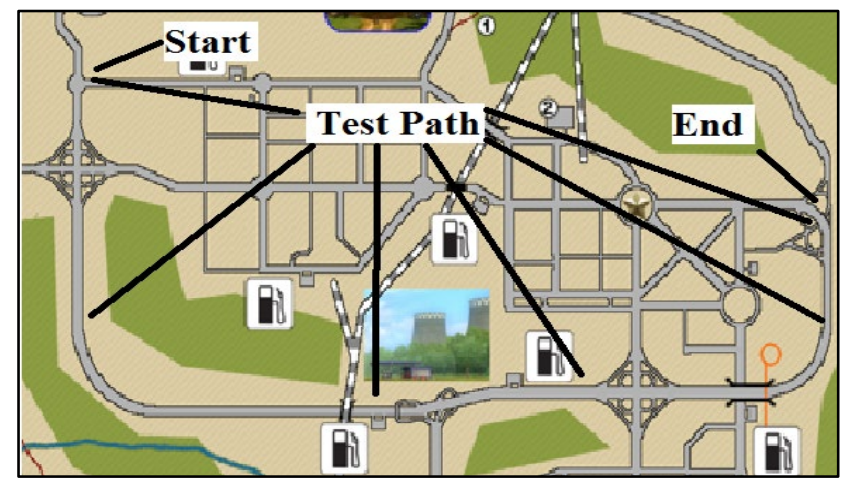

Figure 3: Test path (simulator program screenshot)

\subsubsection{Experimental work and procedure}

In the case of making a hands-free call, the researcher or a person authorized by the researcher made three calls to the participant, and the duration of each call ranged between 45-60 seconds. The participants were asked to stop as soon as they could upon hearing the voice command (stop). Whereas the researcher asked the participant to stop suddenly while he was busy with the phone call and without prior warning. Three cognitive reflexes times were recorded for each participant in three calls, and then their average was set. Repeat the same in the case of a handheld phone call.

In the case of reading the messages, three text messages were sent by the researcher to the participant. These messages contain questions consisting of general knowledge facts. These facts were previously given written on a paper to the participant before the examination, and he was asked to read them. The average number of characters in the message body was 48 . While the participant is busy reading the message, the researcher asked him to stop abruptly with the phrase (stop) and record the perceptual reaction time three times with each message sent by the researcher and take the average reaction time for the participant. In sending the text messages, the participant had to reply to the three text messages received from the researcher. Where he must take out the mobile phone from his pocket, open the phone, read the message sent by the researcher, which is in the form of a question not exceeding 18 characters, and write the answer and send it to the researcher. During this process and without prior warning, the participant will be asked suddenly to stop. The reaction time will be calculated as in the previous cases and the rate will be taken.

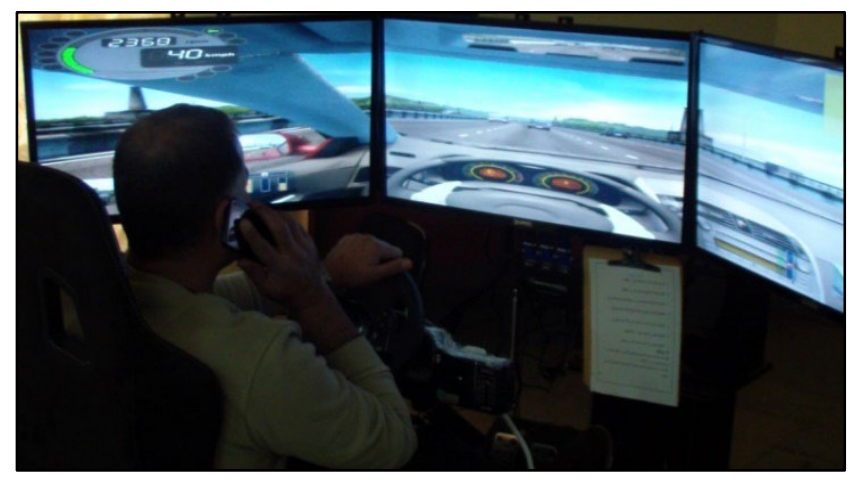

Figure 4: Participant during the test

\section{Results}

\subsection{Mobile Phone Conversations}

\subsubsection{For All Participants}

The results showed a difference in cognitive reaction time between basic driving and engaging in a phone call while driving. In the case of a hands-free phone call, the reaction time increased by about 0.25 seconds, and this gives an impression of being distracted and the mental workload that occurs due to engaging in the phone call. While the increase in response time was higher and clearer in the case of a handheld telephone conversation, where the average reaction time increased from 0.6 
seconds in the case of basic driving to 0.9 seconds while engaging in a handheld phone call with a difference of 0.3 seconds. It is an indication of an increase in mental burden and delay in response resulting from the call on the one hand and from carrying the phone and being busy with it on the other side. These results are consistent with what the researchers concluded[15][16]. See Figure (5).

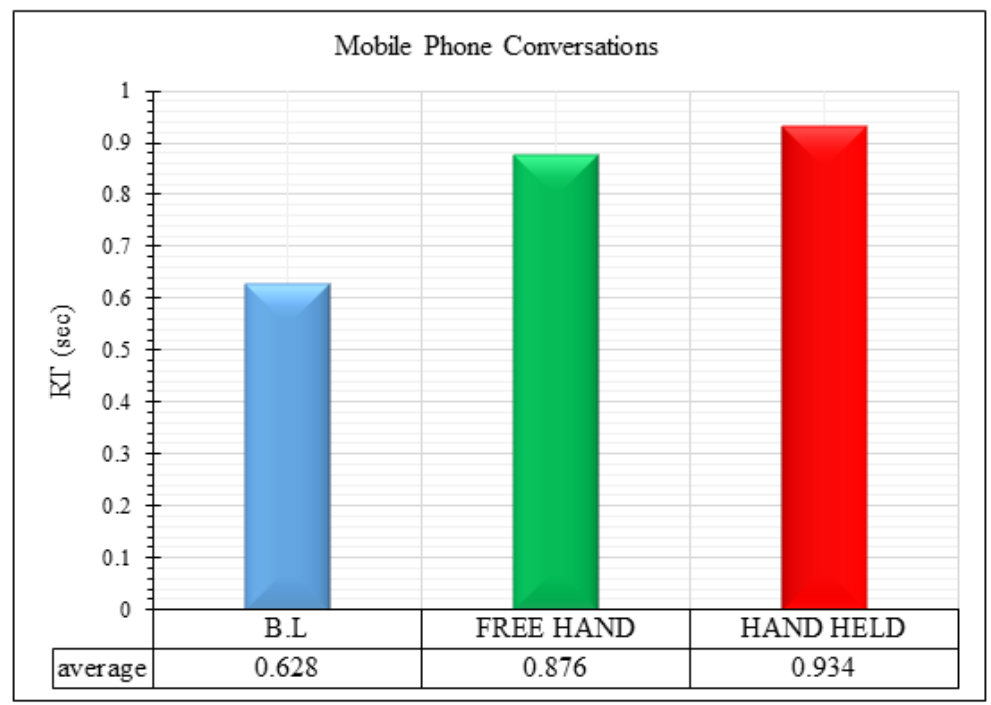

Figure 5: Response time per second by all participants

\subsubsection{By Gender}

The results show a clear effect for both sexes in the cognitive reaction time when they engage in the two communication tasks, whether the phone is hands-free or hand-held. Where the response time for both sexes increased in both tasks, as shown in Figure (6). There were no significant differences in the average response time between gender, except for minor differences, and they were in favor of men, where the response of men was slightly faster. These results are consistent with what the researchers found $[16,17$, and 18].

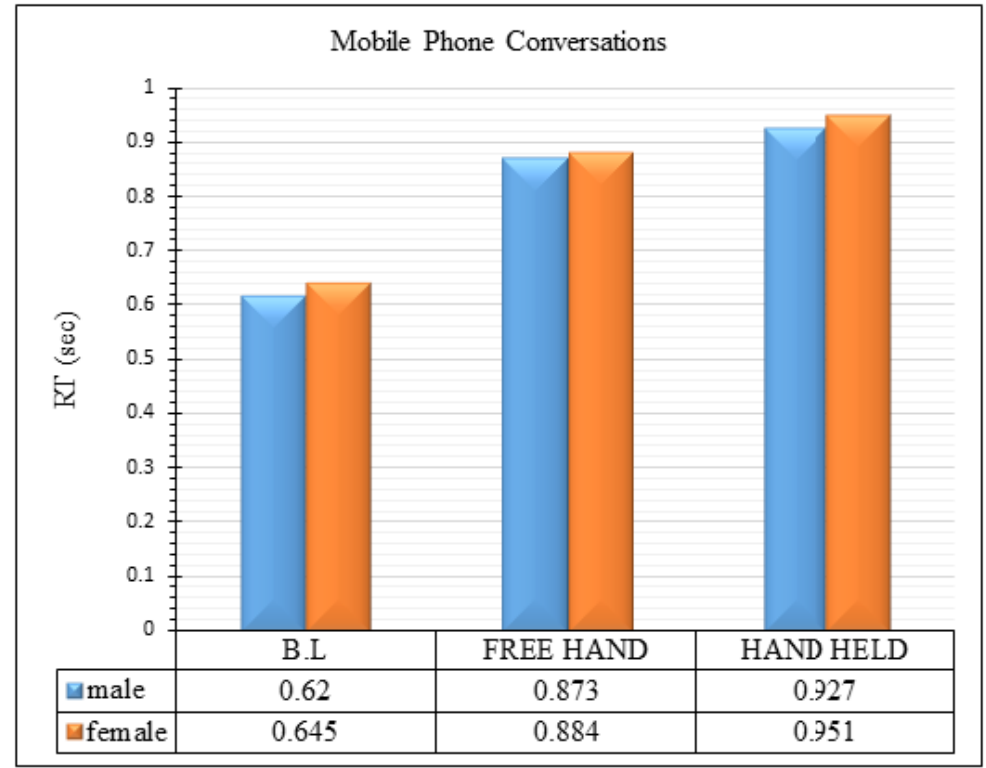

Figure 6: Figure 6: Response time per second by gender

\subsubsection{By Age}

The results obtained from the examination indicate a difference in the response time between the age groups. As shown in Figure (7), we can say that the perceptual reaction time is directly proportional to the age of the participants. The twenty-yearold group recorded the lowest response time in basic driving, while the response time gradually increased with age. In conclusion, the elderly needs more time to respond compared with the younger age groups. Given the perceptual reaction time, while performing the tasks of telephone calls, reaction time has increased for all age groups. An indication of a higher response time while engaging in a handheld telephone conversation with a relatively lower response time in the case of hands-free phone conversations. This can be explained by the fact that the reaction time of perception increases with the difficulty of the 
task that the driver performs while driving, due to its greater mental impact and higher mental and physical effort these results are consistent with the findings of the researcher[19].

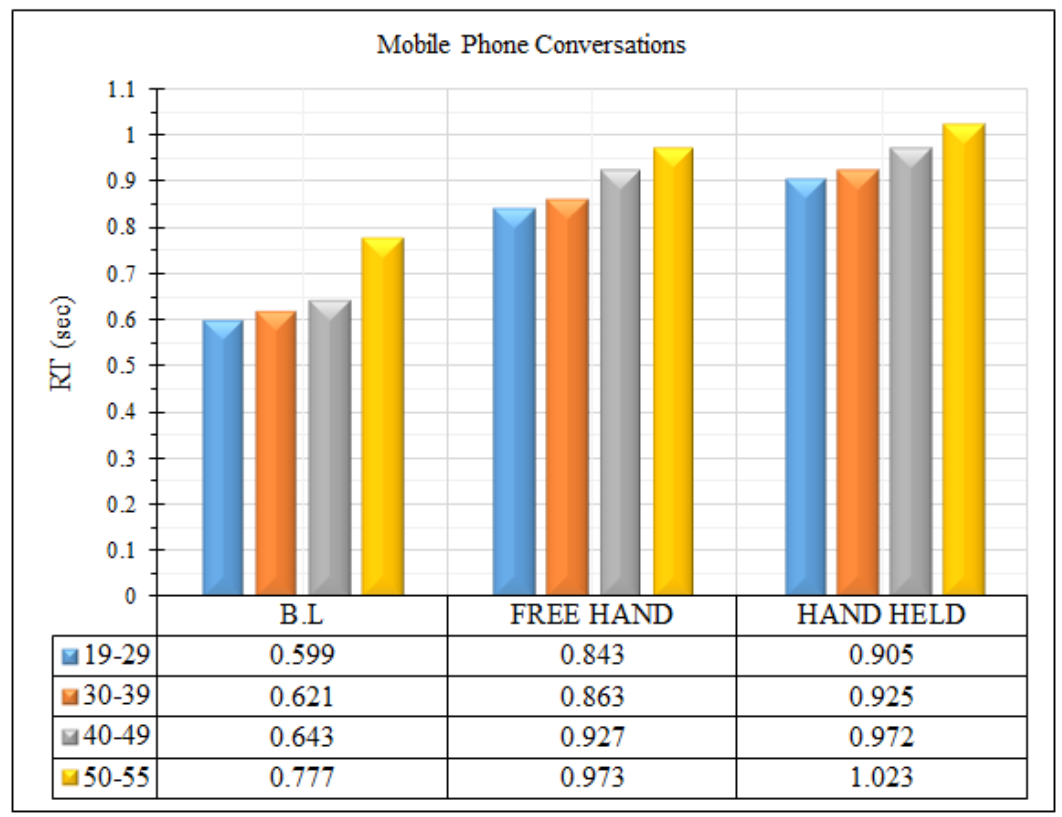

Figure 7: Response time per second by age

\subsection{Text messages}

Text messages contain two tasks, the first is the task of reading incoming messages and answering orally with yes or no. The second task is to respond to an incoming text message. The results obtained from the examination were analyzed based on the total sample, gender, and age groups, and the results were as follows.

\subsubsection{For all Participants}

The results concluded that a clear increase in response time while performing the task of texting while driving for all drivers whose number reached (42) participants. Where the response time in the main leadership was 0.628 seconds, while it increased in the task of reading incoming messages, surpassing the one-second barrier. This can be explained by the fact that drivers have been mentally busy reading the incoming messages, which made the driving task more difficult, and this led to their adaptation to the task situation by reducing the response time. And the matter became more difficult in the task of sending text messages, as the response time increased to almost double than it had been in the case of basic leadership, and this result gives an indication that the response time increases with the increasing difficulty of the task. And because the task of sending the text message requires more mental and physical effort than the rest of the tasks of the mobile phone, whether it is calls or reading messages, so the response time was the highest among them in this task. Figure (8) illustrates the response time between basic leadership and text messaging tasks. This result is consistent with what the researcher concluded[20].

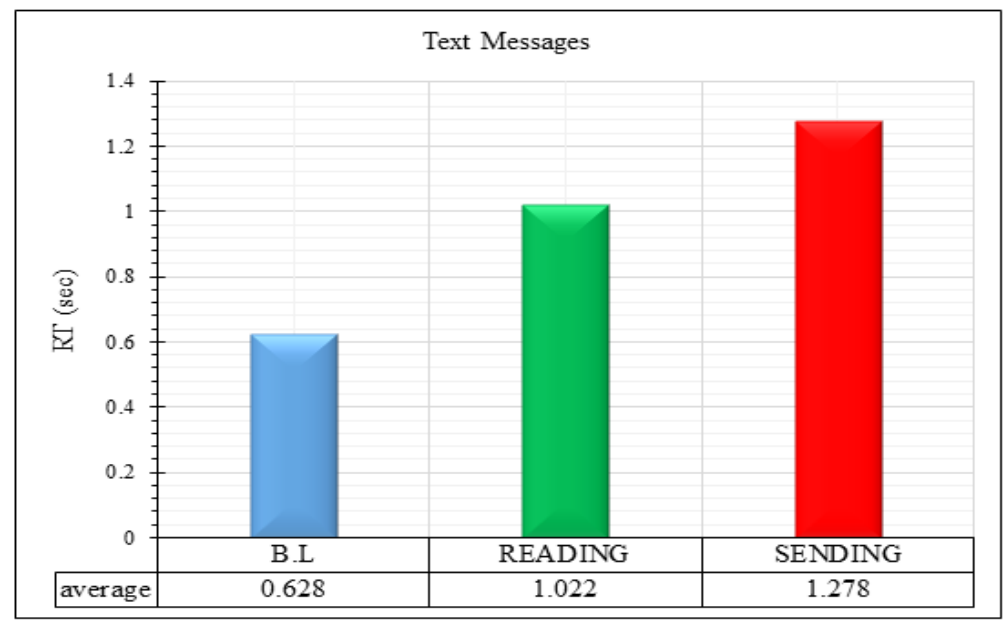

Figure 8: Response time per second by all participants 


\subsubsection{By Gender}

Both genders were affected when performing the two texting tasks. The average response time increased as the task became more difficult. However, no significant differences were recorded between men and women when performing the tasks, except for some differences, which are considered slight. In the case of basic driving, the difference between males and females was 0.022 seconds in favor of men. This is a slight difference. In the task of reading the incoming text messages, the difference between them increased very little, as it was 0.045 seconds. For men, too. In the task of sending text messages, the response rate for men was 0.079 seconds faster than that of women. These results are consistent with the findings of the researchers' mechanism [20, 21]. Figure (9) shows the differences between the sexes while performing text messaging tasks

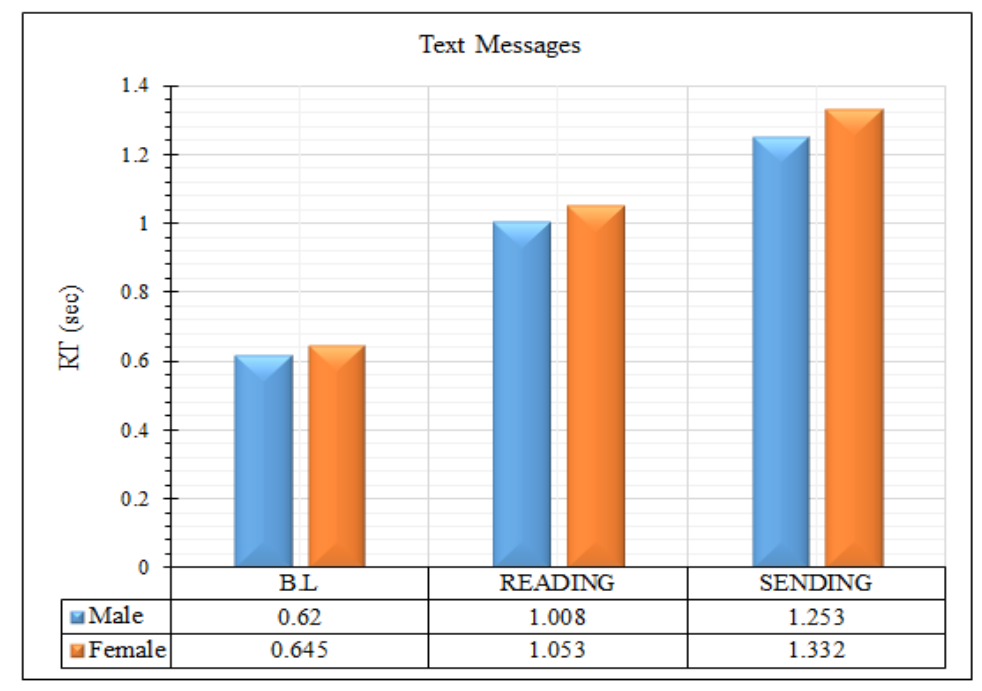

Figure 9: Response time per second by gender

\subsubsection{By Age}

Through the results obtained from the four age groups involved in this study (twenty, thirty, forty, and fifty to fifty-five) years, the differences are evident in the response time, whether it is in the baseline (driving without a task), or in the performance of text messaging tasks. The elderly group (fifties) is the most affected group in performing tasks, as the difference in the task of sending text messages between youth in the twenty years of age and the elderly in the fifties is approximately 0.3 seconds. In either the baseline or driving without a mission, the difference between these two age groups was 0.178 seconds. In general, the performance of text messaging tasks affected all participants of all age groups, but this effect was relative to age. Young age groups in their twenties and thirties were less affected than those in their 40s and 50s.Once again; it appears that the response time is directly proportional to the difficulty of the task and with age. The task of sending text messages had the highest perception reaction time while reading incoming text messages had the lowest response time. Figure (10) shows the differences in the time of cognitive reaction between age groups and texting tasks.

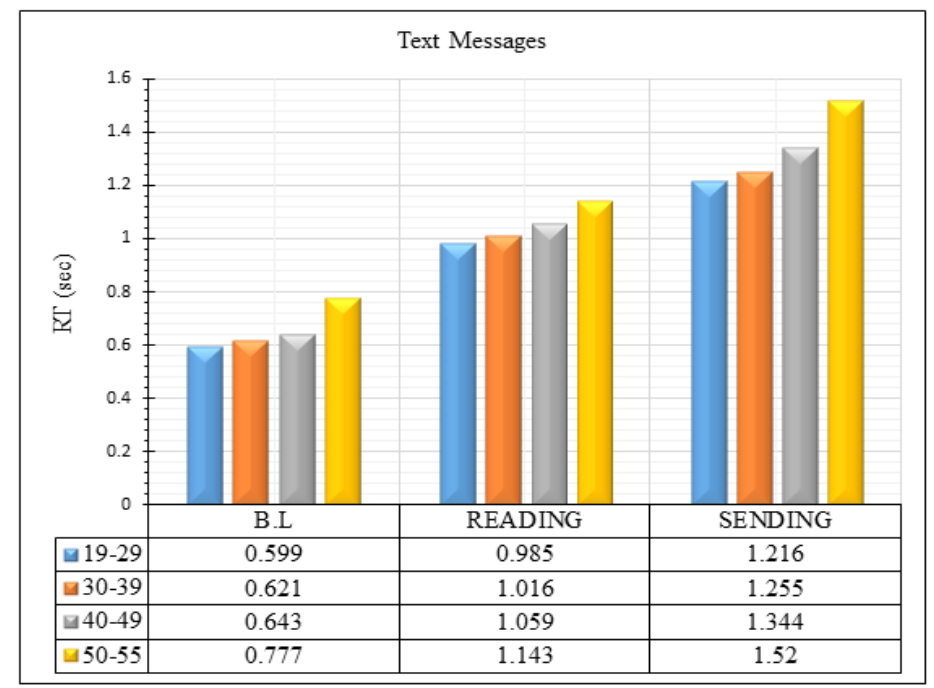

Figure 10: Response time per second by age 


\section{Conclusions}

This study investigated the response time of drivers distracted using a mobile phone in four mobile phone tasks in addition to the basic driving task using a driving simulator. A variety of results were obtained from the 42 participants in this study of different ages and sexes. The participants had to react to the researcher's voice command (stand) and decide to perform the braking maneuver. Response time was calculated from the moment the voice command was issued to the foot lift from the accelerator pedal and the brake pedal. One of the primary factors that explain whether a collision or an accident can be avoided is the reaction parameter[8]. Therefore, intelligent transport systems such as those in vehicles that warn of collision rely mainly on reaction algorithms. Where limits for reaction values ranging from 0.7 to 1.5 seconds are usually used[22]. The results showed that there was a marked increase in the response time of the drivers when they engage in the four phone tasks. This clearly illustrates the danger of using a mobile phone while driving to the public safety of road users. The results indicate that the response time increases with the more difficult the task, as it became clear that the most difficult task is the task of sending text messages, followed by the task of reading incoming text messages, then the task of engaging in a hand-held phone call and finally the task of hands-free phone conversations using headphones. There were no significant differences between the handheld phone calls and the hands-free phone calls, as the difference between them was very slight in terms of the time of the cognitive reaction. In principle, cognitive distraction and distraction are the same between hand-held and hands-free calls. Although the Iraqi Traffic Law No. 3 of 2006 prohibits the use of a mobile phone while driving, and the latest Iraqi law for traffic issued by the Iraqi Council of Representatives and published on 5/8/2019 in the Iraqi newspaper Al-Waif's in Chapter 10 (Penalties) Article 2, Paragraph B prohibits the use of the driver the mobile phone while driving[23], but the law did not mention the details of the prohibition, whether it is forbidden to use it, whether it is hand-held or hands-free. When we asked the traffic officers, they replied that it is customary to punish those who use a hand-held cell phone specifically, and not to punish other forms of use. Therefore, we recommend the legislative bodies take into consideration these results. On the other hand, it's possible that the decreased responsiveness is due to greater competition for focus resources when driving and executing secondary tasks. As the mental as well as the physical burden increases when performing secondary tasks with driving, leading to the dispersion of the drivers.

\section{Acknowledgment} grateful.

In this respect, I cannot help but extend my sincere thanks to everyone who helped us to accomplish this work, and we are

\section{Author contribution}

All authors contributed equally to this work.

\section{Funding}

This research received no specific grant from any funding agency in the public, commercial, or not-for-profit sectors.

\section{Data availability statement}

The data that support the findings of this study are available on request from the corresponding author.

\section{Conflicts of interest}

The authors declare that there is no conflict of interest.

\section{References}

[1] M. Zhu, T. M. Rudisill, K. J. Rauscher, D. M. Davidov, and J. Feng, Risk perceptions of cellphone use while driving: Results from a Delphi survey, Int. J. Environ. Res., 15 (2018). https://doi.org/10.3390/ijerph15061074

[2] Global status report on road safety (2018) summary, (2018). Accessed: Apr. 17, (2021). [Online]. Available:

[3] A. Benedetto, A. Calvi, and F. D'Amico, Effects of mobile telephone tasks on driving performance: A driving simulator study, Adv. Transp. Stud., 41 (2012) 29-44. https://doi.org/10.4399/97888548465863

[4] C. Huisingh, R. Griffin, and G. McGwin, The Prevalence of Distraction Among Passenger Vehicle Drivers: A Roadside Observational Approach Traffic Inj. Prev., 16 (2015) 140-146. https://doi.org/10.1080/15389588.2014.916797

[5] Geneva, A Growing problem of driver distraction 2011 who library cataloguing-in-publication data contentsts, World Heal. Organ.

[6] C. Hallett, A. Lambert, and M. A. Regan, Text messaging amongst New Zealand drivers: Prevalence and risk perception, Transp. Res. Part F Traffic Psychol. Behav., 15 (2012) 261-271. https://doi.org/10.1016/j.trf.2011.12.002

[7] N. C. for S. and Analysis, Distracted driving in fatal crashes, 2017. US Department of Transportation, National Highway Traffic Safety ..., (2019). 
[8] P. Choudhary and N. R. Velaga, Modelling driver distraction effects due to mobile phone use on reaction time, Transp. Res. Part C Emerg. Technol., 77 (2017) 351-365. https://doi.org/10.1016/j.trc.2017.02.007

[9] M. M. Haque and S. Washington, A parametric duration model of the reaction times of drivers distracted by mobile phone conversations, Accid. Anal. Prev., 62 (2014) 42-53. https://doi.org/10.1016/j.aap.2013.09.010

[10] W. Consiglio, P. Driscoll, M. Witte, and W. P. Berg, Effect of cellular telephone conversations and other potential interference on reaction time in a braking response, Accid. Anal. Prev., 35 (2003) $495-500$. https://doi.org/10.1016/S0001-4575(02)00027-1

[11] J. Törnros and A. Bolling, Mobile phone use - effects of conversation on mental workload and driving speed in rural and urban environments, Transp. Res. Part Of Traffic Psychol. Behav., 9(2006) 298-306. https://doi.org/10.1016/j.trf.2006.01.008

[12] A. Subhi, Estimating the passenger car eqaivalent (PCE) for different type of vehicles on the signalized Intersections, Eng. Technol. J., 31 (2013).

[13] A. S. Abdul-Jabbar, Studying Alternatives and Traffic Solutions to Change an Existing Three Legs intersection to an interchange, Eng. Technol. J., 31 (2013). https://doi.org/10.30684/etj.31.2A.15

[14] S. S. Mahmood and L. J. Saud, An efficient approach for detecting and classifying moving vehicles in a video based monitoring system, Eng. Technol. J., 38 (2020) Accessed: May 29, (2021). https://doi.org/10.30684/etj.v38i6A.438

[15] P. Droździel, S. Tarkowski, I. Rybicka, and R. Wrona, Drivers 'reaction time research in the conditions in the real traffic, Open Eng., 10 (2020) 35-47. https://doi.org/10.1515/eng-2020-0004

[16] A. Urs and H. R. Urs, Effect of cellphone conversation and text messaging on driver behaviour: distracted driving,( 2016).

[17] M. F. Lesch and P. A. Hancock, Driving performance during concurrent cell-phone use: Are drivers aware of their performance decrements?, Accid. Anal. Pr., 36 (2004) 471-480. https://doi.org/10.1016/S0001-4575(03)00042-3

[18] I. Spyropoulou and M. Linardou, Modelling the effect of mobile phone use on driving behaviour considering different use modes, J. Adv. Transp., (2019). https://doi.org/10.1155/2019/2196431

[19] R. West, K. J. Murphy, M. L. Armilio, F. I. M. Craik, and D. T. Stuss, Lapses of intention and performance variability reveal age-related increases in fluctuations of executive control ,Brain Cogn., 49 (2002) $402-419$. https://doi.org/10.1006/brcg.2001.1507

[20] J. K. Caird, K. A. Johnston, C. R. Willness, M. Asbridge, and P. Steel, A meta-analysis of the effects of texting on driving, Accid. Anal. Prev., 71 (2014) 311-318. https://doi.org/10.1016/j.aap.2014.06.005

[21] C. V. Oramas, Effect of Cellphone Conversation and Text Messaging on Driver Behaviour: Distracted Driving, (2016).

[22] M. Green, How long does it take to stop?, Methodological Analysis of Driver Perception-Brake Times, Transp. Hum. Factors., 2 (2000) 195-216. https://doi.org/10.1207/sthf0203 1

[23] https://www.itp.gov.iq/ar/qanwn-rqm-8-lsnt-(2019) accessed Apr. 21, (2021) 